Research Paper

International Journal of Biological Sciences

ISSN 1449-2288 www.biolsci.org 2008 4(4):208-214

(C) Ivyspring International Publisher. All rights reserved

\title{
Relatively high levels of serum adiponectin in obese women, a potential indicator of anti-inflammatory dysfunction: Relation to sex hormone- binding globulin
}

\author{
Altan Onat1,2, Gülay Hergenç ${ }^{3}$, Dursun Dursunoğlư ${ }^{4}$, Zekeriya Küçükdurmaz ${ }^{5}$, Serkan Bulur6, Günay Can ${ }^{2}$ \\ 1. Turkish Society of Cardiology, Istanbul University, İstanbul, Turkey \\ 2. Departments of Cardiology and Public Health, Cerrahpaşa Medical Faculty, Istanbul University, İstanbul, Turkey \\ 3. Biology Department, Yildız Technical University, İstanbul, Turkey \\ 4. Department of Cardiology, Pamukkale University Medical Faculty, Denizli, Turkey \\ 5. Department of Cardiology, Gaziantep University Medical Faculty, Gaziantep, Turkey \\ 6. Department of Cardiology, Düzce University Medical Faculty, Düzce, Turkey
}

Correspondence to: Prof.Dr. Altan Onat, Nisbetiye cad. 37/24, Etiler 34335, İstanbul, Turkey. Tel. 902123516217 , Fax 902123514235 , E-mail: alt_onat@yahoo.com.tr

Received: 2008.06.13; Accepted: 2008.07.19; Published: 2008.07.25

It is unclear whether serum adiponectin concentrations diminish linearly with increasing adiposity and, if not, which factors codetermine this association. These issues were investigated cross-sectionally in 1188 men and women, representative of middle-aged and elderly Turkish adults. Serum total adiponectin was assayed by ELISA. Serum adiponectin values in men, though declining significantly in transition from the bottom to the mid tertile of body mass index (BMI) and waist circumference (WC), were similar in the two respective upper tertiles. In women, serum adiponectin concentrations were not significantly different in any tertile of these indices, were significantly correlated with BMI or WC within the low tertiles and not within the two higher tertiles. In a linear regression analysis for WC (or BMI) in a subset of the sample in which serum sex hormone-binding globulin (SHBG) was available and which additionally comprised adiponectin, fasting insulin and other confounders, only insulin and, in women SHBG, were significantly associated, but not adiponectin. In linear regression analyses for covariates of adiponectin in two models comprising 12 variables, insulin and SHBG concentrations were significantly associated in both genders though not BMI. Whereas in men HDL-cholesterol and CRP were covariates of adiponectin (both $\mathrm{p}<0.01)$, SHBG and apolipoprotein B positively associated in women $(\mathrm{p}<0.001)$, independent of BMI and fasting insulin levels.

Conclusions: Relationship between excess adiposity and adiponectin levels is inconsistent in Turkish adults. Independently from obesity and hyperinsulinemia, serum adiponectin discloses significant relationship with inflammatory markers and HDL only in men, not in women in whom it is influenced by SHBG, with consequent attenuation of its anti-inflammatory activities.

Key words: Adiponectin, anti-inflammatory function, gender difference, waist circumference, obesity, sex hormone-binding globulin

\section{Introduction}

In vitro studies and animal experiments have demonstrated that adiponectin, the cytokine synthesized by adipocytes, possesses antiatherogenic, insulin-sensitizing and antiinflammatory properties. In humans, however, conflicting results have been reported between serum adiponectin and coronary heart disease (CHD) [1-3] in one of which [3] was suggested that there may be a true sex difference in the association of adiponectin with coronary heart disease.
Moreover, the theoretical hypothesis of 'adiponectin resistance' was put forward due to the observation of a positive association of plasma adiponectin concentration and age in men, and it was conjectured whether the implication of hypoadiponectinemia in youth might be different from old age [4]. 'Adiponectin paradox' [5], namely a high adiponectin level being a predictor of mortality has been repeatedly observed in patients with chronic heart failure [6], particularly in subjects with normal body mass index (BMI) [6], suggesting that the prognostic significance of adiponectin 
in this condition is affected by BMI. Furthermore, elevated adiponectin levels independently predicted adverse outcomes in 325 male patients presenting with chest pain which was attributed to either the underlying inflammatory state or to adiponectin resistance [7].

Whether the relation of adiponectin concentrations with indices of obesity is of linear shape at increasing degrees of obesity in diverse ethnic groups needs further exploring, particularly as the relation of dyslipidemia with increasing abdominal obesity is of asymptotic shape among Turkish adults, particularly in women $[8,9]$. We recently reported also that sex hormone-binding globulin (SHBG) is an important determinant of dyslipidemia and cardiometabolic disorders, especially in women, independent of abdominal obesity and insulin resistance [10] which warrants the investigation also of the association between SHBG and adiponectin levels.

Thus, the aim of this study was to evaluate: 1) the nature and linearity of the association between different indicators of obesity and serum adiponectin levels, 2) whether or not anticipated inverse associations between adiponectin and HDL-cholesterol, triglycerides/apo B and CRP exist independent of BMI or waist circumference, 3) whether SHBG plays an independent role on concentrations of adiponectin in Turkish men and women in whom both atherogenic dyslipidemia and MetS prevail [11].

\section{Population and Methods}

\section{Sample population}

This study sample was recruited randomly in an approximately three-fifth proportion of participants of the 2005/06 follow-up survey of the Turkish Adult Risk Factor Study, an ongoing study on the prevalence of cardiac disease and risk factors in a representative sample of adults in Turkey carried out periodically almost biennially since 1990 in 59 communities throughout all geographical regions of the country [12]. Details of the overall sampling were described previously [13]. The study was approved by the Ethics Committee of the Istanbul University Medical Faculty. Written informed consent was obtained from all participants. Partial logistic support was provided by the Turkish Ministry of Health. Data were obtained by history of the past years via a questionnaire, physical examination of the cardiovascular system, sampling of blood and recording of a resting 12-lead electrocardiogram. Serum concentrations of adiponectin were assayed among participants aged 37 to 79 years, resulting in determinations in 561 men and 663 women, median age 54 years in each sex, $70 \%$ of whom was fasting. Twenty-two subjects who had serum creatinine concentrations $\geq 133 / 115 \mu \mathrm{mol} / \mathrm{L}$ in men/women, 3 subjects with adiponectin concentrations $>60 \mu \mathrm{g} / \mathrm{ml}$ and 11 persons with missing obesity measures were excluded, leaving 1188 (of whom 644 female) individuals for analysis. This sample did not include any participants who reported suffering from acute inflammatory dlsease in the preceding month. The percentage of postmenopausal women was 65.6. The study group and the whole cohort were similar with respect to age, sex distribution, proportions of metabolic disorders.

\section{Measurement of risk factors}

Blood pressure was measured with an aneroid sphygmomanometer (Erka, Germany) in the sitting position on the right arm, and the mean of two recordings 3 min apart was recorded. Height was measured without shoes using a measuring stick and weight in light indoor clothes using scales. Waist circumference was measured - with the subject standing and wearing only underwear, at the level midway between the lower rib margin and the iliac crest. BMI was computed as weight divided by height squared $\left(\mathrm{kg} / \mathrm{m}^{2}\right)$. In regard to cigarette smoking, nonsmokers, former smokers and current smokers formed the categories.

Blood samples were collected, spun at $1000 \mathrm{~g}$ for 10 minutes and shipped on cooled gel packs at $2-5^{\circ} \mathrm{C}$ to Istanbul to be stored in deep-freeze at $-75^{\circ} \mathrm{C}$, until analyzed at the Yildiz Technical University. Serum concentrations of hsC-reactive protein (CRP), apolipoprotein (apo) B and apo A-I were measured by nephelometry (BN Prospec, Behring Diagnostics, Westwood, MA). Serum concentration of adiponectin was assayed by a sandwich enzyme-linked immunosorbent assay system (Adiponectin ELISA BioVendor, BioVendor Lab. Medicine, Inc, Czech Rep.) at the Acibadem Labmed, Istanbul. Within-run and day-to-day coefficients of variation for adiponectin measurements were $4 \%$ and $<10 \%$, respectively. Serum concentrations of total cholesterol, fasting triglycerides, glucose, HDL-cholesterol (HDL-C plus 2nd generation, directly without precipitation) and LDL-cholesterol (directly) were determined by using enzymatic kits from Roche Diagnostics (Mannheim, Germany) with a Hitachi 902 autoanalyzer. Serum concentrations of SHBG and insulin were carried out by the electrochemiluminescence immunoassay ECLIA on Roche Elecsys 2010 using Roche kits (Roche Diagnostics, Mannheim, Germany). Homeostatic model assessment (HOMA) was calculated with the following formula [14]: insulin $(\mathrm{mIU} / \mathrm{L})^{*}$ glucose (in $\mathrm{mmol} / \mathrm{L}) / 22.5$. 


\section{Data analysis}

Descriptive parameters were shown as mean \pm standard deviation and in percentages. Due to the skewed distribution, values derived from log-transformed (geometric) means and standard error (SE) were used for adiponectin, CRP, insulin, HOMA and SHBG. Pearson correlation tests were made for continuous and log-transformed variables. The population sample was divided into tertiles of BMI (by cutoff points of 25.88 and $29.3 \mathrm{~kg} / \mathrm{m}^{2}$ in men, 27.9 and $32.47 \mathrm{~kg} / \mathrm{m}^{2}$ in women), of WC (by cutoff points of 91 and $101 \mathrm{~cm}$ in men, 87 and $98 \mathrm{~cm}$ in women) and of hip circumference (by 98 and $104 \mathrm{~cm}$ in men, 102 and 112 $\mathrm{cm}$ in women). Pairwise comparisons with Bonferroni adjustments were made to detect significance between groups of estimated means; two-sided t-tests and Pearson's chi-square tests were used to analyze the differences between means and proportions of other groups. Multiple linear regression analyses were performed with continuous parameters, adjusted also for usage of antihypertensive, antidiabetic and lipid lowering drugs, and the presence of CHD. A value of $\mathrm{p}<0.05$ on the two-tail test was considered statistically significant. Statistical analyses were performed using SPSS-10 for Windows.

\section{Results}

This sample of a general population comprised individuals with type- 2 diabetes in $13.2 \%$ and with CHD in $12.4 \%$. Geometric mean value of serum adiponectin concentrations in the entire sample was $9.84 \pm 1.70 \mu \mathrm{g} / \mathrm{ml}$; in the nonfasting state, only $1.6 \%$ lower $(\mathrm{p}=0.49)$ than the $9.89 \pm 1.70 \mu \mathrm{g} / \mathrm{ml}$ among fasting participants. Table 1 shows the distribution of main characteristics of the population sample across tertiles of BMI. Only HDL-cholesterol, triglycerides, apo B, fasting insulin, HOMA index, SHBG, CRP and adiponectin concentrations were significantly different in the bottom tertile (lean individuals) from those in the other tertiles. In contrast, top tertile (obese individuals) differed significantly $(\mathrm{p}<0.01)$ from the mid tertile only in HOMA index and concentrations of insulin and CRP. Significant variables were similarly distributed across tertiles of WC. In this sample with no elevated serum creatinine values, partial correlation coefficients for adiponectin with creatinine, controlled for age, were $-0.13, p=0.003$ in men and $-0.04, p=0.35$ in women. In Table 1, certain variables are presented separately for women as well. These show a similar distribution in the BMI tertiles except for HDL-cholesterol which does not significantly decline in the mid-tertile.

Table 1. Distribution of adiponectin, markers of dyslipidemia and insulin resistance across sex-specific BMI tertiles ( $\mathrm{n}=1188$ ), and separately in women

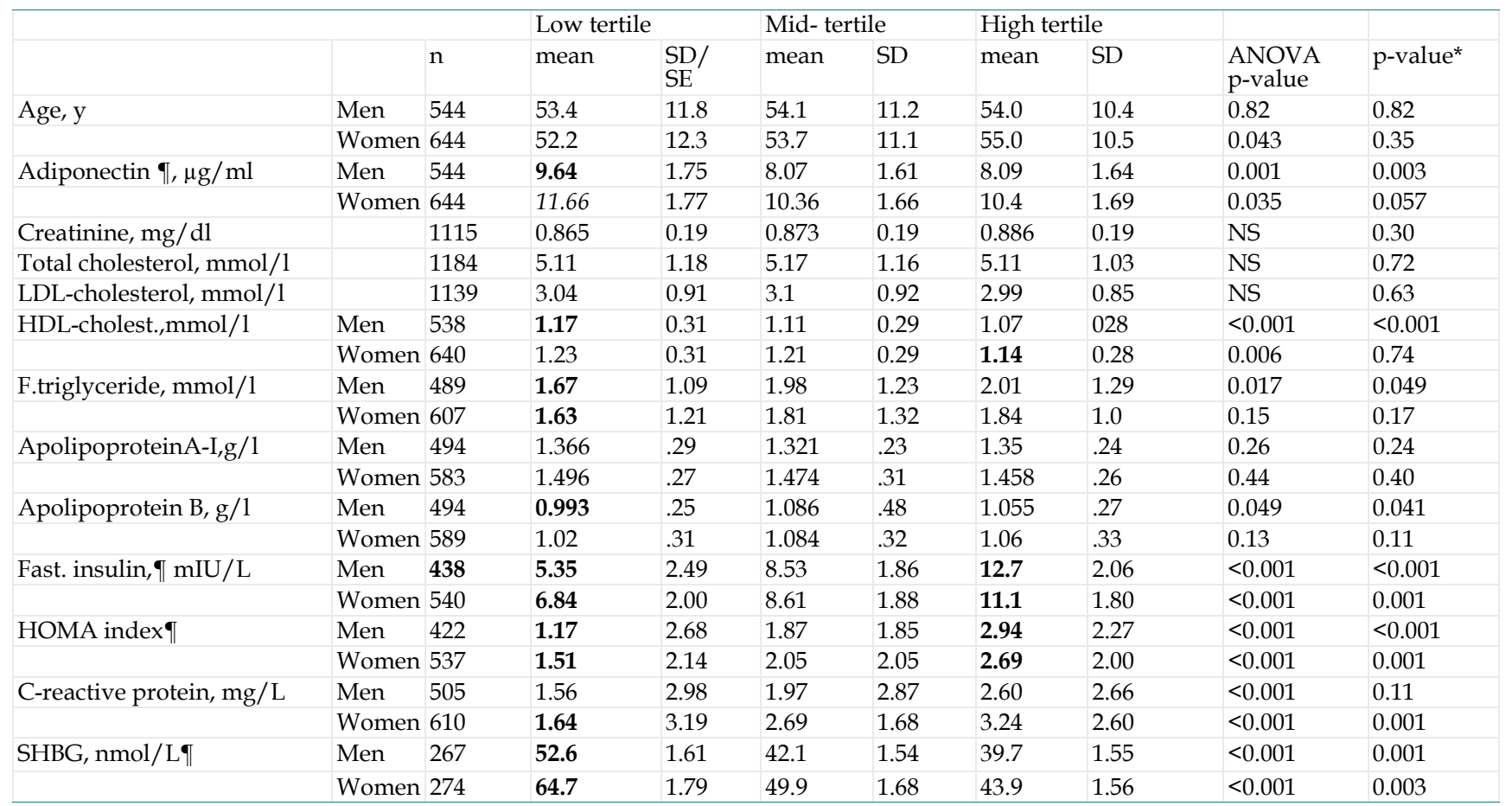

\log-transformed

*between bottom and mid tertiles, significant values indicated in bold 
The distribution of mean adiponectin values as well as their correlation with markers of obesity across tertiles of BMI and WC is provided in Fig. 1, separately in the sexes. Adiponectin concentrations in men were reduced significantly in the mid and top tertiles relative to the bottom tertile, but were similar in the mid and top tertiles of both obesity measures. Adiponectin concentrations among women were similar across tertiles of both obesity indices. Significant inverse correlation between adiponectin and obesity measures was observed only within the low tertiles of BMI but, otherwise, not within the two higher tertiles. Postmenopausal women exhibited essentially the same features as the whole female group.

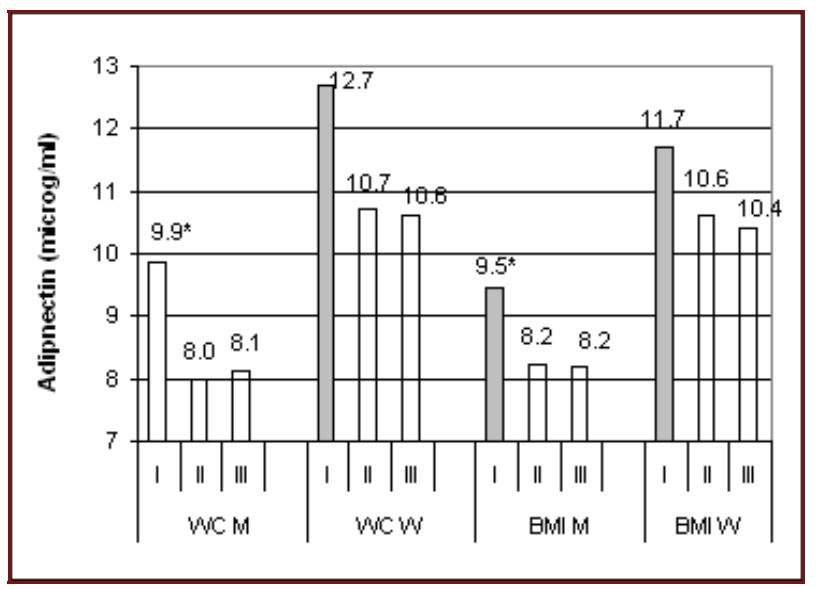

Fig. 1. Mean values of log-transformed adiponectin concentrations stratified by tertiles of waist circumference (WC) and body mass index (BMI) in 544 men and 644 women. Asterisks denote significant $(\mathrm{p}<0.013)$ differences. Shaded bars indicate significant inverse correlation of adiponectin within the related tertile. Mean values of obesity indices in each mid tertile among men and women were as follows: $96-92.5 \mathrm{~cm} ; 27.7-30.2 \mathrm{~kg} / \mathrm{m}^{2}$.

Two regression models were formed seeking linear relationship of serum adiponectin $(\mathrm{p}<0.001)$ which explained $13-20 \%$ of the variance of adiponectin both involving sex, age, insulin, HDL-cholesterol, apo $B$ and SHBG (Table 2). In the first model waist girth and triglycerides were comprised, in the second model BMI tertiles, CRP, prevalent coronary disease and usage of antihypertensive, antidiabetic and lipid lowering drugs. While waist girth or BMI tertiles were not associated, fasting insulin and SHBG were the only significant covariates of adiponectin in both sexes. HDL-cholesterol, apo B and CRP, parameters related to pro- and anti-inflammatory processes, showed different associations in the sexes. Whereas adiponectin revealed anticipated reciprocal associations in males, no association was noted in females in whom adiponectin was positively associated $(\mathrm{p}=0.034)$.
Table 3 shows results of a further linear regression analyses for covariates of serum adiponectin as the dependent variable and including also testosterone, smoking status (not significant), BMI and waist circumference, triglycerides and CRP; as in previous models, availability of SHBG measurement limited the sample to less than half the size. Apart from being determined by female sex $(1.34$-fold, $\mathrm{p}<0.001)$, serum adiponectin was significantly associated not with obesity measures. Inverse association with triglycerides and CRP existed in men, in addition to a positive one with testosterone level (1.23-fold), but in women, only SHBG was associated [B-coefficient 2.07, $\mathrm{p}<0.001$ ].

Table 2. Linear regression for serum adiponectin, $9(\mu \mathrm{g} / \mathrm{ml})$ as dependent variable

\begin{tabular}{|c|c|c|c|c|}
\hline & \multicolumn{2}{|c|}{ Men $\mathrm{n}=229$} & \multicolumn{2}{|c|}{ Women $\mathrm{n}=246$} \\
\hline & B coeff. & p-value & ß coeff. & p-value \\
\hline Fasting insulin, $\uparrow \mathrm{mIU} / 1$ & 0.81 & 0.029 & 0.79 & 0.041 \\
\hline SHBG, $₫ \mathrm{nmol} / 1$ & 1.40 & 0.073 & 2.05 & $<0.001$ \\
\hline HDL-cholesterol, mg/dl & 1.015 & $<0.001$ & 1.001 & 0.62 \\
\hline Apolipoprotein B, mg/l & 1.000 & 0.58 & 1.002 & 0.034 \\
\hline Waist circumference, $\mathrm{cm}$ & 1.00 & 0.95 & 1.001 & 0.65 \\
\hline \multicolumn{5}{|c|}{$\begin{array}{l}\text { Models were significant }(\mathrm{p}<0.001) \text { explaining } 19 \% \text { of variance of } \\
\text { adiponectin in men and } 15 \% \text { in women. Models included also sex } \\
(1.172, \mathrm{p}=0.001) \text {, age and triglycerides, both not significant. }\end{array}$} \\
\hline Fasting insulin, $9 \mathrm{mIU} / 1$ & 0.782 & 0.012 & 0.809 & 0.071 \\
\hline SHBG, $₫ \mathrm{nmol} / 1$ & 1.49 & 0.031 & 2.03 & $<0.001$ \\
\hline HDL-cholesterol, mg/dl & 1.014 & $<0.001$ & 1.002 & 0.53 \\
\hline $\begin{array}{l}\text { C-reactive protein, } \uparrow \\
\mathrm{mg} / 1\end{array}$ & 0.848 & 0.18 & 0.991 & 0.91 \\
\hline BMI tertiles & 1.069 & 0.13 & 1.013 & 0.78 \\
\hline
\end{tabular}

Models were significant $(p<0.001)$ explaining $20 \%$ of variance of adiponectin in men and $13 \%$ in women. Models included also sex $(1.20, p<0.001)$, age, apolipoprotein $B$, usage of antihypertensive, antidiabetic and lipid lowering drugs and prevalent coronary disease, none significant.

Il log-transformed values

The 205 postmenopausal women were not significntly different from the entire female group.

Table 3. Linear regression for serum adiponectin, $9(\mu \mathrm{g} / \mathrm{ml})$ as dependent variable

\begin{tabular}{|c|c|c|c|c|}
\hline & \multicolumn{2}{|c|}{ Men $\mathrm{n}=244$} & \multicolumn{2}{|c|}{ Women $\mathrm{n}=264$} \\
\hline & B coeff. & $\mathrm{p}$-value & B coeff. & p-value \\
\hline Testosterone, $\mathbf{~ n m o l / 1 ~}$ & 1.23 & 0.041 & 1.008 & 0.92 \\
\hline $\mathrm{SHBG}, \boldsymbol{\Upsilon} \mathrm{nmol} / \mathrm{l}$ & 1.46 & 0.051 & 2.07 & $<0.001$ \\
\hline F. triglycerides, $\mathrm{mg} / \mathrm{dl}$ & 0.9994 & 0.042 & 1.000 & 0.28 \\
\hline $\begin{array}{l}\text { C-reactive protein, } \\
\mathrm{mg} / \mathrm{l}\end{array}$ & 0.824 & 0.051 & 0.994 & 0.92 \\
\hline
\end{tabular}

Models were significant $(\mathrm{p}<0.001)$ explaining $18 \%$ of variance of adiponectin in men and $10 \%$ in women. Models included also sex (1.34, $\mathrm{p}=0.001)$, age ( $\mathrm{p}=0.012$ in men), smoking status, BMI and waist circumference, none significant.

If log-transformed values

\section{Discussion}

In this cross-sectional study on middle-aged and elderly Turkish adults, we have shown that serum adiponectin was not linearly associated with measures 
of overall or abdominal obesity when controlled for insulin and SHBG. In fact, except for lean subjects, there was a total lack of association of obesity measures with adiponectin. Serum adiponectin values displayed a threshold effect with increasing obesity, beyond which concentrations did not significantly decline. In multivariable linear analyses, adiponectin levels were significantly associated with HDL-cholesterol and CRP, independent of BMI and fasting insulin levels only in men while being in women positively associated with apo B, an inflammatory marker [15], and with SHBG. These three novel findings, namely, a threshold effect, evidence of a gender difference in anti-inflammatory properties of adiponectin and an independent association of low SHBG with moderately low adiponectin levels in women may lead to the generation of a hypothesis for women that adiponectin possesses impaired anti-inflammatory properties, likely influenced by SHBG levels, while the emergence of an inverse relationship between obesity and adiponectin levels is precluded.

\section{Lack of inverse association between obesity indices and} serum adiponectin among obese persons

It is generally considered that plasma adiponectin levels decrease with increasing obesity. A salient finding of the present analysis is the lack of further change of adiponectin values in the transition to high tertiles of obesity measures beyond the mid tertile, regardless of genders. This suggests an impaired relationship of adiponectin with adiposity in this population. An inverse association could not be demonstrated in multiple linear regression analyses between adiponectin and measure(s) of (abdominal) obesity in either gender. This is in partial agreement with the recent work of Yasui et al. [16] in which serum adiponectin concentrations in postmenopausal women alone were neither correlated, nor associated with BMI independently of SHBG but were so among men.

Reports on the role of lower body fat mass on adiponectin have yielded conflicting results. An independent positive role, partly explained by variations of adiponectin level, was observed in 401 men by Buemann et al [17], and subcutaneous adipose tissue was a positive independent correlate of adiponectin in nondiabetic Americans [18]. A significant correlation between $\mathrm{HC}$ and adiponectin was, however, lacking in 165 Asian Indian and Caucasian adults living in Canada [19], and adiponectin was significantly inversely correlated with subcutaneous abdominal adipose tissue in 148 women [20] or in 41 Caucasian men by computed tomography [21]. Taken together, these findings show a lack of a linear association of adi- ponectin with (abdominal) obesity measures, and levels fail to be reduced in response to increasing adiposity.

Synthesis and secretion of adiponectin by adipocytes, or interaction with specific adiponectin receptors might be impaired in obese individuals. A state of adiponectin resistance was recently described in insulin resistant mice with hyperadiponectinemia with normal levels of adiponectin receptor- 1 and -2 and inability to lower glucose levels by adiponectin administration [22]. In nonobese patients with chronic heart failure, high serum adiponectin paradoxically predicts mortality $[5,6]$; it is unclear whether the anti-inflammatory property of this protein is impaired under these conditions.

\section{Independent association of SHBG with adiponectin concentrations}

Our finding of testosterone being significantly associated with serum adiponectin is in line with the report of Nishizawa and coworkers [23] that androgens, being suppresed with aging, decrease plasma adiponectin. The independent association of adiponectin with SHBG has been sparsely investigated. In 334 elderly Japanese men and women, SHBG was found to be a determinant of adiponectin in multivariate analysis in both genders, regardles of HOMA index, and in men alone, also independent of BMI [17]. Our multivariable analyses in women, by demonstrating that SHBG is independently and inversely related to WC as well as to adiponectin levels, irrespective of obesity measures, insulin or CRP levels or of menopausal status, suggest that relatively low levels may be heterogenous and, indeed, be linked to adiponectin functional defectiveness. This observation supports our recent report that low SHBG levels significantly associate, particularly in women, with various metabolic disorders, independent of abdominal obesity and insulin resistance [10] and suggests that this effect is mediated by adiponectin.

\section{Gender difference possibly affecting adiponectin's anti-inflammatory function}

Present findings indicate that expected associations of adiponectin concentrations with HDL-cholesterol and CRP in men is independent of variations in SHBG and other relevant parameters such as obesity, insulin level and smoking status. This is in essential agreement with the recent report on the association of adiponectin in Japanese men with CRP independently of all MetS factors [24]. At distinct variance, in Turkish women, no independent positive relationship of adiponectin levels was observed with HDL-cholesterol, nor a reciprocal one with CRP, in fact, a positive association emerged with apo B, e.g. 
adiponectin depended on variations in SHBG and insulin, regardless of inflammatory markers. This suggests a concept that, the anti-inflammatory activities of adiponectin counteract two types of inflammatory processes, one related to obesity/insulin resistance, opposed by but not independently linked to adiponectin, the other not related to obesity/insulin resistance (rather perhaps to lipoproteins, hormones, chemokine attractant protein, adhesion molecules) and opposed by adiponectin. The latter type of anti-inflammatory properties manifested herein by significant independent associations between adiponectin levels and CRP or HDL among men. In females of this sample, evidence of such dysfunction was observed. Impaired interaction with one of the known or unknown adiponectin receptors enhanced by low SHBG might be hypothesized as an underlying mechanism, perhaps with resulting interaction between adiponectin and apo B. It has been pointed out that SNPs for adiponectin receptor-1 gene were significant in obese but not in combined lean and obese subjects, suggesting gene-environment interactions, such that polymorphisms may influence obesity-associated comorbidities only when individual is obese [25].

This gender difference very likely explains the observation that low SHBG is an important determinant of both diabetes and the dyslipidemia involving hypertriglyceridemia with elevated apo B in women (though not in men) independent of abdominal obesity and insulin resistance [10]. In studying a large sample of patients with coronary heart disease, van Eynatten et al. [26] found a lack of association of adiponectin with CRP which they attributed to a potential decrease of the role of systemic inflammation as part of the relationship of adiponectin with atherosclerosis during the course of the disease.

Further gender-related dysfunction of protective proteins have been newly observed in this sample of the adult Turkish population at large. As yet unpublished observations provided evidence of anti-inflammatory and atheroprotective HDL dysfunction as well as indicated that high compared with low serum apo A-I levels independently increase the risk for incident diabetes. Collectively, these alterations suggest that under circumstances of excess chronic low-grade inflammation associated with a high prevalence of obesity and cardiometabolic diseases, several defense mechanisms such as activities of circulating protective proteins may be seriously attenuated.

The main limitation in this study is its cross-sectional nature with its attendant potential inability to differentiate cause from effect. Though no significant difference was found between adiponectin concentrations of fasting and nonfasting subjects, a previously described slight diurnal variation in concentrations may not have been accounted in the current analyses. Nonetheless, it is difficult to dispute that present findings provide convincing evidence of a lack of anti-inflammatory properties of adiponectin and a potential role of low SHBG in Turkish women therein. The study's large sample size, being based on a representative sample of a general population of both sexes, in which MetS prevailed highly, the range of obesity measures were wide, adjustments were made for such parameters as insulin, CRP, apo B and SHBG and validity shown also exclusively in menopausal women constitute strengths of the study. Results warrant exploration and confirmation in other ethnic populations.

In conclusion, we found that circulating adiponectin, not linearly associated with measures of overall or abdominal obesity, disclosed a lack of (independent) association with obesity measures and a threshold effect. Adiponectin failed to be linked with CRP and HDL and was positively associated with apo $B$ in women, contrasted to men. Serum SHBG was significantly and independently associated with serum adiponectin levels among women. It may be hypothesized that low SHBG in obese women is independently related to generation of functionally defective adiponectin, which might not afford enough protection against inflammatory processes, obscuring the manifestation of an inverse relationship between obesity and adiponectin levels. Gender determines an independence of such partial anti-inflammatory dysfunction of adiponectin from obesity. These observations implicate that moderately high adiponectin values in obese individuals should not be interpreted as necessarily indicating low cardiometabolic risk; they may further provide insight into the strong inflammatory component underlying cardiometabolic risk in diverse ethnic populations.

\section{Supplementary Material}

\section{Supplementary Table [http://www.biolsci.org/v04p0208s1.pdf]}

\section{Acknowledgements}

We thank the Turkish Society of Cardiology and SanofiAventis, İstanbul, as well as the pharmaceutical companies AstraZeneca, Novartis and Pfizer, İstanbul, Turkey, that have supported financially the Turkish Adult Risk Factor survey 2005/06. We are indebted to I. Unsal, MD, S. Gögüş and M. Menekşe for the ELISA assays of serum adiponectin. We appreciate the dedicated works of S. Albayrak, MD, A. Karabulut, MD, 
A.M. Esen, MD, and Mr. M. Özmay, the coworkers in the survey teams.

\section{Conflict of Interest}

The authors have declared that no conflict of interest exists.

\section{References}

1. Pischon T, Girman CJ, Hotamisligil GS, Rifai N, Hu FB,Rimm EB. Plasma adiponectin levels and risk of myocardial infarction in men. JAMA 2004;291:1730-7

2. Schulze MB, Shai I, Rimm EB, Li T, Rifai N, Hu FB. Adiponectin and future coronary heart disease events among men with type 2 diabetes. Diabetes 2005;54:534-9

3. Lawlor DA, Davey Smith G, Ebrahim S, Thompson C, Satar N. Plasma adiponectin levels are associated with insulin resistance, but do not predict coronary heart disease risk in women. J Clin Endocrinol Metab 2005;90:5677-83

4. Iwashima Y, Katsuya T, Ishikawa K, et al. Hypoadiponectinemia is an independent risk factor for hypertension. Hypertension 2004;43:1318-23

5. Tsutamoto T, Tanaka T, Sakai H, Ishikawa C, Fujii M, Yamamoto $\mathrm{T}$, Horie M. Total and high molecular weight adiponectin, haemodynamics, and mortality in patients with chronic heart failure. Eur Heart J 2007;28:1723-30

6. Kistorp C, Faber J, Galatius S, Gustafsson F, Frystyk J, Flyvbjerg A, Hildebrandt P. Plasma adiponectin, body mass index, and mortality in patients with chronic heart failure. Circulation 2005;112:1756-62

7. Cavusoglu E, Ruwende C, Chopra V, et al. Adiponectin is an independent predictor of all-cause mortality, cardiac mortality, and myocardial infarction in patients presenting with chest pain. Eur Heart J 2007;27:2300-9

8. Onat A, Sarı I, Hergenç G, Yazıcı M, Can G, Uyarel H, Sansoy V. Predictors of abdominal obesity and high susceptibility of cardiometabolic risk to its increments among Turkish women: a prospective population-based study. Metabolism 2007;56:348-56

9. Onat A, Uyarel H, Hergenç G, Karabulut A, Albayrak S, Can G. Determinants and definition of abdominal obesity as related to risk of diabetes, metabolic syndrome and coronary disease in Turkish men: a prospective cohort study. Atherosclerosis 2007;191:182-90

10. Onat A, Hergenç G, Karabulut A, Albayrak S, Can G, Kaya Z. Serum sex hormone-binding globulin, a determinant of cardiometabolic disorders independent of abdominal obesity and insulin resistance in elderly men and women. Metabolism 2007;56:1356-62

11. Onat A, Ceyhan K, Başar Ö, Erer B, Toprak S, Sansoy V. Metabolic syndrome: major impact on coronary risk in a population with low cholesterol levels - a prospective and cross-sectional evaluation. Atherosclerosis 2002;165:285-92

12. Onat A. Risk factors and cardiovascular disease in Turkey. Atherosclerosis 2001;156:1-10.

13. Onat A, Avcı GŞ, Şenocak M, Örnek E, Gözükara Y. Plasma lipids and their interrelation in Turkish adults. J Epidem Commun Health 1992;46:470-476.

14. Mather KJ, Hunt AE, Steinberg HO, et al. Repeatability characteristics of simple indices of insulin resistance: implications for research applications. J Clin Endocrinol Metab 2001;86:5457-64.

15. Onat A, Can G, Hergenç G, Yazıcı M, Karabulut A, Albayrak S. Serum apolipoprotein B predicts dyslipidemia, metabolic syndrome and, in women, hypertension, diabetes, independent of markers of central obesity and inflammation. Int J Obes 2007; 31:1119-25
16. Yasui T, Tomita J, Miyatani Y, et al. Associations of adiponectin with sex hormone-binding globulin levels in aging male and female populations. Clin Chim Acta 2007;386:69-75

17. Buemann B, Sörensen TIA, Pedersen O, et al. Lower-body fat mass as an independent marker of insulin sensitivity - the role of adiponectin. Int J Obes 2005;29:624-31

18. Hanley AJ, Bowden D, Wagenknecht LE, et al. Associations of adiponectin with body fat distribution and insulin sensitivity in nondiabetic Hispanics and African-Americans. J Clin Endocrinol Metab 2007;82:2665-71

19. Smith J, Al-Amri M, Sniderman A, Cianflone K. Leptin and adiponectin in relation to body fat percentage, waist to hip ratio and the apoB/apoAI ratio in Asian Indian and Caucasian men and women. Nutrition Metabol 2006;3:18

20. Ryan AS, Borman DM, Nicklas BJ, et al. Plasma adiponectin and leptin levels, body composition, and glucose utilization in adult women with wide ranges of obesity. Diabetes Care 2003;26:2383-8

21. Farvid MS, Ng TWK, Chan DC, Barrett PHR, Watts GF. Association of adiponectin and resistin with adipose tissue compartments, insulin resistance and dyslipidaemia. Diabetes Obes Metabol 2005;7:406-13

22. Lin HV, Kim JY, Pocai A., Rossetti L, Shapiro L, Scherer PE, Acilci D. Adiponectin resistance exacerbates insulin resistance in insulin receptor transgenic/knockout mice. Diabetes 2007;56:1969-76

23. Nishizawa H, Shimomura I, Kishida K, et al. Androgens decrease plasma adiponectin, an insulin-sensitizing adipocyte-derived protein. Diabetes 2002;51:2734-41

24. Matsushita K, Tamakoshi K, Yatsuya H, et al. Further inflammatory information on metabolic syndrome by adiponectin evaluation. Int J Cardiol 2008; 124:339-44

25. Crimmins NA, Martin LJ. Polymorphisms in adiponectin receptor genes ADIPOR1 and ADIPOR2 and insulin resistance. Obes Rev 2007;8:419-23

26. Von Eynatten M, Hamann A, Twardella D, Nawroth PP, Brenner $\mathrm{H}$, Rothenbacher D. Relationship of adiponectin with markers of systemic inflammation, atherogenic dyslipidemia, and heart failure in patients with coronary heart disease. Clin Chem 2006;52:853-9 